\title{
On resistive magnetohydrodynamic equilibria of an axisymmetric toroidal plasma with flow
}

\author{
G. N. Throumoulopoulos H and H. Tasso \\ Max-Planck-Institut für Plasmaphysik, EURATOM Association \\ D-85748 Garching, Germany
}

October 1999

\begin{abstract}
It is shown that the magnetohydrodynamic equilibrium states of an axisymmetric toroidal plasma with finite resistivity and flows parallel to the magnetic field are governed by a second-order partial differential equation for the poloidal magnetic flux function $\psi$ coupled with a Bernoulli type equation for the plasma density (which are identical in form to the corresponding ideal MHD equilibrium equations) along with the relation $\Delta^{\star} \psi=V_{c} \sigma$. (Here, $\Delta^{\star}$ is the Grad-SchlüterShafranov operator, $\sigma$ is the conductivity and $V_{c}$ is the constant toroidal-loop voltage divided by $2 \pi$ ). In particular, for incompressible flows the above mentioned partial differential equation becomes elliptic and decouples from the Bernoulli equation [H. Tasso and G. N. Throumoulopoulos, Phys. Plasmas 5, 2378 (1998)]. For a conductivity of the form $\sigma=\sigma(R, \psi)$ ( $R$ is the distance from the axis of symmetry) several classes of analytic equilibria with incompressible flows can be constructed having qualitatively plausible $\sigma$ profiles, i.e. profiles with $\sigma$ taking a maximum value close to the magnetic axis and a minimum value on the plasma surface. For $\sigma=\sigma(\psi)$ consideration of the relation $\Delta^{\star} \psi=V_{c} \sigma(\psi)$ in the vicinity of the magnetic axis leads therein to a proof of the non-existence of either compressible or incompressible equilibria. This result can be extended to the more general case of non-parallel flows lying within the magnetic surfaces.

PACS numbers: 52.30.Bt, 52.55.-s
\end{abstract}

\footnotetext{
${ }^{1}$ Permanent address: University of Ioannina, Association EURATOM / Hellenic Republic, Physics Department, Section of Theoretical Physics, GR 45110 Ioannina, Greece
} 


\section{Introduction}

In addition to the case of the long living astrophysical plasmas, understanding the equilibrium properties of resistive fusion plasmas is important, particularly in view of the next step devices which will possibly demand pulse lengths of the order of $10^{3}$ secs (or more for an ITER size machine) ([1] and Refs. cited therein). Theoretically, however, it was proved by Tasso [2] that resistive equilibria with $\sigma=\sigma(\psi)$ are not compatible with the Grad-Schlüter-Shafranov equation. (Here, $\sigma$ is the conductivity and $\psi$ is the poloidal magnetic flux function.) The nonexistence of static axisymmetric resistive equilibria with a uniform conductivity

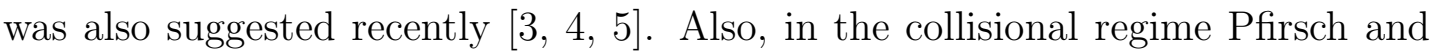
Schlüter showed that the toroidal curvature gives rise to an enhanced diffusion, which is related to the conductivity parallel to the magnetic field. In the above mentioned studies the inertial-force flow term $\rho(\mathbf{v} \cdot \nabla) \mathbf{v}$ is neglected in the equation of momentum conservation. For ion flow velocities of the order of $100 \mathrm{Km} / \mathrm{m}$, which have been observed in neutral-beam-heating experiments [6, [7, 8] the term $\rho(\mathbf{v} \cdot \nabla) \mathbf{v}$ can not be considered negligible. Therefore, it is worthwhile to investigate the nonlinear resistive equilibrium, in particular to address the following issues: (a) the impact of the non-linear flow in the Pfirsch-Schlüter diffusion, and (b) the existence of resistive equilibria, in particular equilibria with $\sigma=\sigma(\psi)$. Since the magnetohydrodynamic (MHD) equilibrium with arbitrary flows and finite conductivity is a very difficult problem, in a recent study [9] we considered an axisymmetric toroidal plasma with purely toroidal flow including the term $\rho(\mathbf{v} \cdot \nabla) \mathbf{v}$ in the momentum-conservation equation. It was shown that the nonlinear flow does not affect the static-equilibrium situation, i.e $\sigma=\sigma(\psi)$ equilibria are not possible.

A way of constructing more plausible equilibria from the physical point of view could be by considering flows less restricted in direction. Taking also into account the fact that the poloidal flow in the edge region of magnetic-confinement systems plays a role in the transition from the low-confinement mode to the highconfinement mode, in the present report we extend our previous studies to the case of flows having non-vanishing poloidal components in addition to toroidal ones. Because of the difficulty of the problem we consider flows parallel to the magnetic field. Some of the conclusions, however, can be extended to non-parallel flows lying within the magnetic surfaces. It is also noted that possible equilibria with 
parallel flows would be free of Pfirsch-Schlüter diffusion because the convective term $\mathbf{v} \times \mathbf{B}$ in the Ohm's low vanishes. The main conclusion is that for the system under consideration the existence of equilibria depends crucially on the spatial dependence of conductivity. The report is organized as follows. The equilibrium equations for an axisymmetric toroidal resistive plasma with parallel flows surrounded by a conductor are derived in Sec. II. The existence of solutions is then examined in Sec. III for the cases $\sigma=\sigma(R, \psi)$ ( $R$ is the distance from the axis of symmetry), and $\sigma=\sigma(\psi)$. Sec. IV summarizes our conclusions.

\section{Equilibrium equations}

The MHD equilibrium states of a plasma with scalar conductivity are governed by the following set of equations, written in standard notations and convenient units:

$$
\begin{gathered}
\nabla \cdot(\rho \mathbf{v})=0, \\
\rho(\mathbf{v} \cdot \nabla) \mathbf{v}=\mathbf{j} \times \mathbf{B}-\nabla P, \\
\nabla \times \mathbf{E}=0, \\
\nabla \times \mathbf{B}=\mathbf{j}, \\
\nabla \cdot \mathbf{B}=0, \\
\mathbf{E}+\mathbf{v} \times \mathbf{B}=\frac{\mathbf{j}}{\sigma} .
\end{gathered}
$$

It is pointed out that, unlike to the usual procedure followed in equilibrium studies with flow [10, 11, 12, 13, 14, 15] in the present work an equation of state is not included in the above set of equations from the outset and therefore the equation of state independent Eqs. (15) and (16) below are first derived. This alternative procedure is convenient because the equilibrium problem is then further reduced for specific cases associated with several equations of state.

The system under consideration is a toroidal axisymmetric magnetically confined plasma, which is surrounded by a conductor (see Fig. 1 of Ref. [9]). With the use of cylindrical coordinates $R, \phi, z$ the position of the surface of the conductor is specified by some boundary curve in the $(R, z)$ plane. The equilibrium quantities do not depend on the azimuthal coordinate $\phi$. Consequently, the divergence free magnetic field $\mathbf{B}$ and current density $\mathbf{j}$ can be expressed, with the 
aid of Ampere's low (四), in terms of the stream functions $\psi(R, z)$ and $I(R, z)$ as

$$
\mathbf{B}=I \nabla \phi+\nabla \phi \times \nabla \psi,
$$

and

$$
\mathbf{j}=\Delta^{\star} \psi \nabla \phi-\nabla \phi \times \nabla I .
$$

Here, $\Delta^{\star}$ is the elliptic operator defined by $\Delta^{\star}=R^{2} \nabla \cdot\left(\nabla / R^{2}\right)$ and constant $\psi$ surfaces are magnetic surfaces. Also, it is assumed that the plasma elements flow solely along $\mathbf{B}$ :

$$
\rho \mathbf{v}=K \mathbf{B},
$$

where $K$ is a function of $R$ and $z$. Acting the divergence operator on Eq. (9) and taking into account Eq. (11) one obtains $\nabla K \cdot \mathbf{B}=0$. Therefore, the function $K$ is a surface quantity:

$$
K=K(\psi) .
$$

Another surface quantity is identified from the toroidal component of the momentum conservation equation (2):

$$
\left(1-\frac{K^{2}}{\rho}\right) I=X(\psi) .
$$

From Eq. (11) it follows that, unlike the case in static equilibria, I is not (in general) a surface quantity. Furthermore, expressing the time independent electric field by

$$
\mathbf{E}=-\nabla \Phi+V_{c} \nabla \phi,
$$

where $V_{c}$ is the constant toroidal-loop voltage divided by $2 \pi$, the poloidal and toroidal components of Ohm's law (6), respectively, yield

$$
\nabla \Phi=\frac{\nabla \phi \times \nabla I}{\sigma}
$$

and

$$
\Delta^{\star} \psi=V_{c} \sigma=E_{\phi} R \sigma .
$$

Here, $E_{\phi}$ is the toroidal component of $\mathbf{E}$. Eq. (14) has an impact on the boundary conditions, i.e. the component of $\mathbf{E}$ tangential to the plasma-conductor interface does not vanish. Therefore, the container can not be considered perfectly conducting. Accordingly, Ohm's law with finite conductivity applied in the vicinity 
of the plasma-conductor interface does not permit the existence of a surface layer of current [16]. It is now assumed that the position of the conductor is such that its surface coincides with the outermost of the closed magnetic surfaces. Thus, the condition $\mathbf{B} \cdot \mathbf{n}=0$, where $\mathbf{n}$ is the outward unit vector normal to the plasma surface, holds in the plasma-conductor interface and therefore the pressure $\mathrm{P}$ must vanish on the boundary. It is noticed that this is possible only in equilibrium, because in the framework of resistive MHD time dependent equations, the magnetic flux is not conserved. With the aid of equations (7)-(11) the components of Eq. (2) along $\mathbf{B}$ and perpendicular to a magnetic surface are put in the respective forms

$$
\text { B } \cdot\left[\nabla\left(\frac{K^{2} B^{2}}{2 \rho^{2}}\right)+\frac{\nabla P}{\rho}\right]=0
$$

and

$$
\begin{array}{r}
\left\{\nabla \cdot\left[\left(1-\frac{K^{2}}{\rho}\right) \frac{\nabla \psi}{R^{2}}\right]+\frac{K}{\rho} \frac{\nabla K \cdot \nabla \psi}{R^{2}}\right\}|\nabla \psi|^{2} \\
+\left\{\rho \nabla\left(\frac{K^{2} B^{2}}{2 \rho^{2}}\right)+\frac{\nabla I^{2}}{2 R^{2}}-\frac{\rho}{2 R^{2}} \nabla\left(\frac{I K}{\rho}\right)^{2}+\nabla P\right\} \cdot \nabla \psi=0 .
\end{array}
$$

Eq. (16) has a singularity when

$$
\frac{K^{2}}{\rho}=1 \text {. }
$$

On the basis of Eq. (9) for $\rho \mathbf{v}$ and the definitions $v_{A p}^{2} \equiv \frac{|\nabla \psi|^{2}}{\rho}$ for the Alfvén velocity associated with the poloidal magnetic field and the Mach number

$$
M^{2} \equiv \frac{v_{p}^{2}}{v_{A p}^{2}}=\frac{K^{2}}{\rho},
$$

Eq. (17) can be written as $M^{2}=1$.

Summarizing, the resistive MHD equilibrium of an axisymmetric toroidal plasma with parallel flow is governed by the set of Eqs. (14), (15) and (16). Owing to the direction of the flow parallel to B, Eqs. (15) and (16) do not contain the conductivity and are identical in form to the corresponding equations governing ideal equilibria. Therefore, on the one hand, several properties of the ideal equilibria, e.g. the Shafranov shift of the magnetic surfaces and the detachment of the isobaric surfaces from the magnetic surfaces (see the discussion 
following Eq. (26) in Sec IIC) remain valid. On the other hand, as will be shown in Sec. III, the conductivity $\sigma$ in Eq. (14) plays an important role on the existence of equilibria.

To reduce further equations (15) and (16), the starting set of equations (10)-(河) must be supplemented by an equation of state, e.g. $P=P(\rho, T)$, along with an equation determining the transport of internal energy. Such a rigorous treatment, however, makes the equilibrium problem very cumbersome. Alternatively, one can assume additional properties for the magnetic surfaces associated with either isentropic processes, or isothermal processes, or incompressible flows. These three cases are separately examined in the remainder of this section.

\section{A. Isentropic magnetic surfaces}

We consider a plasma with large but finite conductivity such that for times short compared with the diffusion time scale, the dissipative term $\approx j^{2} / \sigma$ can be neglected. This permits one to assume conservation of the entropy: $\mathbf{v} \cdot \nabla \mathbf{S}=\mathbf{0}$, which on account of Eq. (9) leads to $S=S(\psi)$ ( $S$ is the specific entropy). It is noted that the case $S=S(\psi)$ was considered in investigations on ideal equilibria with arbitrary flows [11, 12] and purely toroidal flows [17, 18], as well as on resistive equilibria with purely toroidal flows [9]. In addition, the plasma is assumed to being a perfect gas whose internal energy density $W$ is simply proportional to the temperature. Then, the equations for the thermodynamic potentials lead to 17

$$
P=A(S) \rho^{\gamma}
$$

and

$$
W=\frac{A(S)}{\gamma-1} \rho^{\gamma-1}=\frac{H}{\gamma} .
$$

Here, $A=A(S)$ is an arbitrary function of $S, H=W+P / \rho$ is the specific enthalpy and $\gamma$ is the ratio of specific heats. For simplicity and without loss of generality we choose the function $A$ to be identical with $S$. Consequently, integration of Eq. (15) yields

$$
\frac{K^{2} B^{2}}{2 \rho^{2}}+\frac{\gamma}{\gamma-1} S \rho^{\gamma-1}=H(\psi)
$$

Eq. (16) reduces then to

$$
\nabla \cdot\left[\left(1-\frac{K^{2}}{\rho}\right) \frac{\nabla \psi}{R^{2}}\right]+(\mathbf{v} \cdot \mathbf{B}) K^{\prime}+\frac{B_{\phi}}{R} X^{\prime}+\rho H^{\prime}-\rho^{\gamma} S^{\prime}=0,
$$


where the prime denotes differentiation with respect to $\psi$. Apart from a factor $1 /(\gamma-1)$ in the last term of the right-hand side $\left([1 /(\gamma-1)] \rho^{\gamma} S^{\prime}\right.$ instead of $\left.\rho^{\gamma} S^{\prime}\right)$ Eq. (22) is identical in form with the corresponding ideal MHD equation obtained by Hameiri [12] (Eq. (7) therein). It should be noted that Eq. (22) remains regular for the case of isothermal plasmas $(\gamma=1)$ while Hameiri's result would make the equilibrium equation strangely singular. In particular, for $S=S(\psi)$ and $T=$ const. Eq. (19) leads to $\rho=\rho(\psi)$ and consequently the incompressibility equation $\nabla \cdot \mathbf{v}=0$ follows from Eq. (11). Incompressible flows, however, are described by Eq. (27) below which is free of the above mentioned singularity.

Unlike the case of static equilibria, Eq. (22) is not always elliptic; there are three critical values of the poloidal-flow Mach-number $M^{2}$ at which the type of this equation changes, i.e. it becomes alternatively elliptic and hyperbolic [10, 12]. The toroidal flow is not involved in these transitions because this is incompressible by axisymmetry and, therefore, does not relate to hyperbolicity (see also the discussion in the beginning of Sec. IIC).

\section{B. Isothermal magnetic surfaces}

Since for fusion plasmas the thermal conduction along $\mathbf{B}$ is expected to be fast in relation to the heat transport perpendicular to a magnetic surface, equilibria with isothermal magnetic surfaces are a reasonable approximation [17, 18, 19, 20, 21, 22]. In particular, the even simpler case of isothermal resistive equilibria has also been considered [23].

For $T=T(\psi)$ integration of Eq. (15) leads to

$$
\frac{K^{2} B^{2}}{2 \rho^{2}}+\lambda T \ln \rho=H(\psi)
$$

where $\lambda$ is the proportionality constant in the ideal gas law $P=\lambda \rho T$. Consequently, Eq. (16) reduces to

$$
\nabla \cdot\left[\left(1-\frac{K^{2}}{\rho}\right) \frac{\nabla \psi}{R^{2}}\right]+(\mathbf{v} \cdot \mathbf{B}) K^{\prime}+\frac{B_{\phi}}{R} X^{\prime}+\rho H^{\prime}-\lambda \rho(1-\log \rho) T^{\prime}=0 .
$$

We remark that apart from the fact that the $\mathrm{S}$ terms have been replaced by $\mathrm{T}$ terms, Eqs. (23) and (24) are identical with the respective Eqs. (21) and (22).

\section{Incompressible flows}


The existence of hyperbolic regimes may be dangerous for plasma confinement because they are associated with shock waves which can cause equilibrium degradation. In this respect incompressible flows are of particular interest because, as is well known from gas dynamics, it is the compressibility that can give rise to shock waves; thus for incompressible flows the equilibrium equation becomes always elliptic. For $\nabla \cdot \mathbf{v}=0$ it follows from Eqs. (1) and (9) that the density is a surface quantity

$$
\rho=\rho(\psi)
$$

consistent with the fact that in fusion experiments equilibrium density gradients parallel to $\mathbf{B}$ have not been observed.

With the aid of Eq. (25), integration of Eq. (15) yields an expression for the pressure:

$$
P=P_{s}(\psi)-\frac{v^{2}}{2}=P_{s}-\frac{K^{2} B^{2}}{2 \rho}
$$

We note here that, unlike in static equilibria, in the presence of flow magnetic surfaces in general do not coincide with isobaric surfaces because Eq. (2) implies that $\mathbf{B} \cdot \nabla P$ in general differs from zero. In this respect, the term $P_{s}(\psi)$ is the static part of the pressure which does not vanish when $\mathbf{v}=\mathbf{0}$. If it is now assumed that $\frac{K^{2}}{\rho} \neq 1$ and Eq. (26) is inserted into Eq. (16), the latter reduces to the elliptic differential equation

$$
\left(1-M^{2}\right) \Delta^{\star} \psi-\frac{1}{2}\left(M^{2}\right)^{\prime}|\nabla \psi|^{2}+\frac{1}{2}\left(\frac{X^{2}}{1-M^{2}}\right)^{\prime}+R^{2} P_{s}^{\prime}=0 .
$$

Eq. (27) is identical in form to the corresponding ideal equilibrium equation (Eq. (22) of Ref. [22]). It is also noted that special cases of incompressible ideal equilibria have been investigated in Refs. [24] and [25]. Unlike to the corresponding sets of compressible $S=S(\psi)$ equations (21) and (22), and $T=$ $T(\psi)$ equations (23) and (24), Eq. (27) is decoupled from Eq. (26). Once the solutions of Eq. (27) are known, Eq. (26) only determines the pressure.

\section{The existence of solutions in relation to the conductivity profile}

We shall show that the compatibility of Eq. (14) containing the conductivity $\sigma$ with the "ideal" equations (15) and (16) depends crucially on the spatial dependence of $\sigma$. In this respect the cases $\sigma=\sigma(R, \psi)$, and $\sigma=\sigma(\psi)$ are examined below. 


$$
\text { A. } \sigma=\sigma(R, \psi)
$$

An explicit spatial dependence of $\sigma$, in addition to that of $\psi$, is interesting because it makes the equilibrium problem well posed, i.e. in this case Eq. (14) can be decoupled from the other Eqs. (15) and (16). A possible explicit spatial dependence of $\sigma$ can be justified by the following arguments: (a) Even in Spitzer conductivity, $\sigma=\alpha T_{e}^{3 / 2}$, the quantity $\alpha$ has a (weak) spatial dependence and (b) cylindrically symmetric resistive $\sigma=\sigma(\psi)$ equilibria are possible [9] and therefore the non-existence of axisymmetric static toroidal $\sigma=\sigma(\psi)$ equilibria is related to the toroidicity involving through the scale factor $|\nabla \phi|=1 / R$; this could also imply an explicit dependence of $\sigma$ on $R$. In addition, we may remark that the neoclassical conductivity depends on the aspect ratio $\mathcal{A}$ because the fraction of trapped particles relates to $\mathcal{A}$ (see [26] and Refs. cited therein). It should be noted, however, that a knowledge of the $\sigma$-profile in the various collisionality regimes of magnetic confinement has not been obtained to date.

For us the main advantage in allowing $\sigma=\sigma(R, \psi)$ lies in the fact that Eq. (14) can then be considered as a formula determining the conductivity

$$
\sigma=\frac{\Delta^{\star} \psi}{V_{c}}
$$

provided $\psi$ is known. Also, the poloidal electric field can then be obtained by Eq. (13).

To determine $\psi$ in the case of compressible flows with isentropic magnetic surfaces the set of Eqs. (21) and (22), which are coupled through the density $\rho$, should be solved numerically under appropriate boundary conditions. This can be accomplished by the existing ideal MHD equilibrium codes [13, 14, 15]. The problem of compressible flows with isothermal magnetic surfaces [Eqs. (23) and (24)] can be solved in a similar way.

For incompressible flows $\psi$ can be determined by Eq. (27) alone, which is amendable to several classes of analytic solutions. In particular, sheared- poloidalflow equilibria associated with "radial" (poloidal) electric fields which play a role in the L-H transition can be constructed by means of the transformation [27, 28]

$$
U(\psi)=\int_{0}^{\psi}\left[1-M^{2}\left(\psi^{\prime}\right)^{1 / 2}\right] d \psi, \quad M^{2}<1,
$$


Under this transformation Eq. (27) reduces (after dividing by $\left.\left(1-M^{2}\right)^{1 / 2}\right)$ to

$$
\Delta^{\star} U+\frac{1}{2} \frac{d}{d U}\left(\frac{X^{2}}{1-M^{2}}\right)+R^{2} \frac{d P_{s}}{d U}=0 .
$$

It is noted here that the requirement $M^{2}<1$ in transformation (29) implies that $v_{p}^{2}<v_{s}^{2}$, where $v_{s}=(\gamma P / \rho)^{1 / 2}$ is the sound speed. This follows from Eqs. (18) and (in Gaussian units)

$$
\left(\frac{v_{s}}{v_{A p}}\right)^{2}=(\gamma / 2) \frac{8 \pi P}{h^{2}|\nabla \psi|^{2}} \approx 1 .
$$

Since, according to experimental evidence in tokamaks [29], the (maximum) value of the ion poloidal velocity in the edge region during the $\mathrm{L}-\mathrm{H}$ transition is of the order of $10 \mathrm{Km} / \mathrm{sec}$ and the ion temperature is of the order of $1 \mathrm{KeV}$, the scaling $v_{p} \ll v_{s}$ is satisfied in this region. Therefore, the restriction $M^{2}<1$ is of nonoperational relevance. The simplest solution of Eq. (27) corresponding to $M^{2}=$ const., $X^{2}=$ const. and $P_{s} \propto \psi$, is given by

$$
\psi=\psi_{c}\left(\frac{R}{R_{c}}\right)^{2}\left[2-\left(\frac{R}{R_{c}}\right)^{2}-d^{2}\left(\frac{z}{R_{c}}\right)^{2}\right],
$$

where $\psi_{c}$ is the $\psi$ value on the magnetic axis located at $\left(z=0, R=R_{c}\right)$ and $d$ is a parameter related to the shape of flux surfaces. Equation (31) describes the Hill's vortex configuration [30]. The conductivity then follows from Eq. (28):

$$
\sigma=\sigma_{c}\left(\frac{R}{R_{c}}\right)^{4}\left[2-\left(\frac{R}{R_{c}}\right)^{2}-d^{2}\left(\frac{z}{R_{c}}\right)^{2}\right],
$$

where $\sigma_{c}$ is the value of $\sigma$ on the magnetic axis. The conductivity profile in the middle-plane $z=0$ is illustrated in Fig. 1. We remark the outward displacement of the maximum-conductivity position $R_{\max }$ with respect to $R_{c}\left(R_{\max } / R_{c}=\right.$ $2 / \sqrt{3}$ ) and the asymetry of the inner part of the profile as compared with the outer part due to the explicit $R$ dependence of $\sigma$.

$$
\text { B. } \sigma=\sigma(\psi)
$$

For this case we consider Eq. (14) in the vicinity of the magnetic axis by transforming the coordinates from $(R, z, \phi)$ to $(x, y, \phi)$ (Fig. 2). The transformation is given by

$$
\begin{aligned}
R & =R_{c}+x=R_{c}+r \cos \theta \\
z & =y=-r \sin \theta
\end{aligned}
$$


The quantities $\psi(x, y)$ and $\sigma(\psi)$ are then expanded to second-order in $x$ and $y$ :

$$
\psi(x, \psi)=\psi_{c}+c_{1} \frac{x^{2}}{2}+c_{2} \frac{y^{2}}{2}+c_{3} x y+\ldots
$$

and

$$
\sigma=\sigma_{c}+\sigma_{1}\left(\psi-\psi_{c}\right)+\ldots=\sigma_{c}+\sigma_{1}\left(c_{1} \frac{x^{2}}{2}+c_{2} \frac{y^{2}}{2}+c_{3} x y+\ldots\right)+\ldots
$$

Here, $c_{1}=\left(\partial^{2} \psi / \partial x^{2}\right)_{c}, c_{2}=\left(\partial^{2} \psi / \partial y^{2}\right)_{c}, c_{3}=\left(\partial^{2} \psi / \partial x \partial y\right)_{c}, \sigma_{c}$ is the conductivity on the magnetic axis and $\sigma_{1}=$ const. On the basis of Eqs. (34) and (35) Eq. $\Delta^{\star} \psi=V_{c} \sigma(\psi)$ becomes a polynomial in $x$ and $y$ which should vanish identically. This requirement leads to $c_{1}=c_{3}=0$ and, therefore, it follows from Eq. (34) that the magnetic surfaces in the vicinity of the magnetic axis are not closed surfaces.

The non-existence of $\sigma(\psi)$ equilibria with closed magnetic surfaces can be extended to the case of non-parallel flows lying within the magnetic surfaces. Indeed, if the relation $\mathbf{v} \cdot \nabla \psi=0$ is assumed instead of $\mathbf{v} \| \mathbf{B}$, the toroidal component of Eq. (6) leads again to Eq. (14).

A possible proof of the non-existence of $\eta=\eta(\psi)$ equilibria far from the magnetic axis has not been obtained to date. It may be noted, however, that for $\sigma=\sigma(\psi)$, Eq. (16) becomes parabolic. This follows by considering in this equation the determinant $\mathcal{D}$ of the symmetric matrix of coefficients. On account of $\Delta^{\star} \psi=V_{c} \sigma(\psi)$, and $\rho=\rho(R, \psi,|\nabla \psi|)$ by Eq. (15), the second derivatives of equation (16) are contained only in the term

$$
\frac{K^{2}}{\rho} \frac{\partial \rho}{\partial|\nabla \psi|^{2}} \nabla|\nabla \psi|^{2} \cdot \nabla \psi
$$

which comes from the term $\nabla \cdot\left[\left(1-K^{2} / \rho\right) \nabla \psi / R^{2}\right]$. Subsequent evaluation of $\mathcal{D}$ leads to $\mathcal{D}=0$. Therefore, the function $\psi$ is (over)restricted everywhere to satisfy a parabolic equation and the elliptic equation $\Delta^{\star} \psi=V_{c} \sigma(\psi)$.

\section{Conclusions}

The equilibrium of an axisymmetric plasma with flow parallel to the magnetic field has been investigated within the framework of the resistive magnetohydrodynamic (MHD) theory. For the system under consideration the equilibrium equations 
reduce to a set of a second-order differential equation for the poloidal magnetic flux function $\psi$ coupled through the density with an algebraic Bernoulli equation, which are identical in form with the corresponding ideal MHD equations, and the equation $\Delta^{\star} \psi=V_{c} \sigma$. ( $\left(\Delta^{\star}, V_{c}\right.$ and $\sigma$ are the Grad-Schlüter-Shafranov elliptic operator, the constant toroidal loop voltage and the conductivity, respectively. The existence of solutions of the above mentioned set of equations is sensitive to the spatial dependence of $\sigma$.

For a conductivity of the form $\sigma=\sigma(R, \psi)$, Eq. $\Delta^{\star} \psi=V_{c} \sigma$ can be considered uncoupled to the other two equations, thus determining only the conductivity. For compressible flows and isentopic magnetic surfaces the differential equation for $\psi$ [(Eq. (22)], pending on the value of the poloidal flow, can be either elliptic or hyperbolic. Solutions of the set of this equation and the coupled Bernoulli equation [Eq. (21)] can be obtained numerically. The problem of compressible equilibria with isothermal magnetic surfaces [Eqs. (23) and (24)] can be solved in a similar way. For incompressible equilibria $\psi$ obeys an elliptic differential equation [(Eq. (27)], uncoupled to the associated Bernoulli equation [Eq. (26)] which just determines the pressure. Several classes of analytic equilibria with incompressible flows having qualitatively plausible $\sigma$ profiles, i.e, profiles with $\sigma$ taking a maximum value close to the magnetic axis and a minimum value on the plasma surface, can be constructed. In particular, sheared-poloidal-flow equilibria can be derived by means of the transformation (29) for $\psi$.

For $\sigma=\sigma(\psi)$ appreciation of $\Delta^{\star} \psi=V_{c} \sigma$ in the vicinity of the magnetic axis proves therein, irrespective of plasma compressibility, the non-existence of closed magnetic surfaces. This result can be extended to the case of non-parallel flows lying within the magnetic surfaces. In addition, for parallel flows $\psi$ is (over)restricted to satisfy throughout the plasma an elliptic and a parabolic differential equations.

According to the results of the present investigation, the existence of resistive equilibria is sensitive to the spatial dependence of conductivity. Thus, the task of obtaining this dependence in the various confinement regimes of fusion plasmas may deserve further experimental and theoretical investigations. A conductivity with a spatial dependence in addition to that of $\psi$, on the one hand, would open up the possibility of the existence of several classes of resistive equilibria free of Pfirsch-Schlüter diffusion. On the other hand, a strict Spitzer-like conductivity, 
$\sigma=\sigma(\psi)$, should imply the persistence of a Pfirsch-Schlüter-like diffusion also in the non-linear flow regime.

\section{Acknowledgments}

Part of this work was conducted during a visit by one of the authors (G.N.T.) to the Max-Planck Institut für Plasmaphysik, Garching. The hospitality of that Institute is greatly appreciated.

\section{References}

[1] D. Moreau and I. Voitsekhovitch, Nucl. Fusion 39, 685 (1999).

[2] H. Tasso, Lectures on Plasma Physics, Report IFUSP/P-181, LFP-8, Universidade de São Paulo, Instituto de Física, São Paulo (1979).

[3] D. Montgomery, and X. Shan, Comments Plasma Phys. Contolled Fusion 15, 315 (1994).

[4] J. W. Bates and H. R. Lewis, Phys. Plasmas 32395 (1996).

[5] D. Montgomery, J. W. Bates, and H. R. Lewis, Phys. Plasmas 4, 1080 (1997).

[6] S. Suckewer, H. P. Eubank, G. J. Goldston E. Hinnov and N. R. Sauthoff, Phys. Rev. Lett. 43, 207 (1979).

[7] K. Brau, M. Bitter, R. J. Goldston, D. Manos K. McGuire, S. Suckewer, Nucl. Fusion 23, 1643 (1983).

[8] H. F. Tammen, A. J. H. Donné, H. Euringer and T. Oyevaar, Phys. Rev. Lett. 72, 356 (1994).

[9] G. N. Throumoulopoulos, J. Plasma Physics 59, 303 (1998).

[10] H. P. Zehrfeld and B. J. Green, Nucl. Fusion 12, 569 (1972).

[11] A. I. Morozov and L. S. Solovév, Reviews of Plasma Physics 8, 1 (1980), edited by M. A. Leontovich (Consultants Bureau, New York).

[12] E. Hameiri, Phys. Fluids 26, 230 (1983). 
[13] S. Semenzato, R. Gruber and H. P. Zehrfeld, Comput. Phys. Rep. 1, 389 (1984).

[14] W. Kerner, and S. Tokuda, Z. Naturforsch. 42a, 1154 (1987)

[15] R. Żelazny, R. Stankiewicz, A. Galkowski and S. Potempski et al., Plasma Phys. Contr. Fusion 35, 1215 (1993).

[16] J. D. Jackson Classical Electrodynamics, Second Edition (John Wiley \& Sons, New York, 1975) p. 335.

[17] E. K. Maschke and H. Perrin, Plasma Phys. 22, 579 (1980).

[18] G. N. Throumoulopoulos and G. Pantis, Phys. Plasmas B 1, 1827 (1989).

[19] R. A. Clemente and R. Farengo, Phys. Fluids 27, 776 (1984).

[20] H. Tasso, Phys. Lett. A 222, 97 (1996).

[21] G. N. Throumoulopoulos and H. Tasso, Phys. Plasmas 4, 1492 (1997).

[22] H. Tasso and G. N. Throumoulopoulos, Phys. Plasmas 5, 2378 (1998).

[23] H. Grad and J. Hogan, Phys. Rev. Lett. 24, 1337 (1970).

[24] K. Avinash, S. N. Bhattacharyya and B. J. Green, Plasma Phys. Control. Fusion 34, 465 (1992).

[25] Zh. N. Andruschenko, O. K. Cheremnykh and J. W. Edenstrasser, J. Plasma Physics 58, 421 (1997).

[26] O. Sauter, C. Angioni and Y. R. Lin-Liu, Phys. Plasmas 6, 2834 (1999).

[27] R. A. Clemente, Nucl. Fusion 33, 963 (1993).

[28] P. J. Morrison, Private communication; transformation (29) was discussed in the invited talk entitled "A generalized energy principle" which was delivered in the Plasma-Physics APS Conference, Baltimore 1986.

[29] K. H. Burrell, Phys. Plasmas 4, 1499 (1997).

[30] W. B. Thompson, An introduction to Plasma Physics (Addison-Wesley, Reading, Massachusetts, 1964), p. 55. 


\section{Figure captions}

FIG. 1. The conductivity profile on the middle-plane $z=0$ described by Eq. (32)

FIG. 2. The system of coordinates $(x, y, \phi)$. 


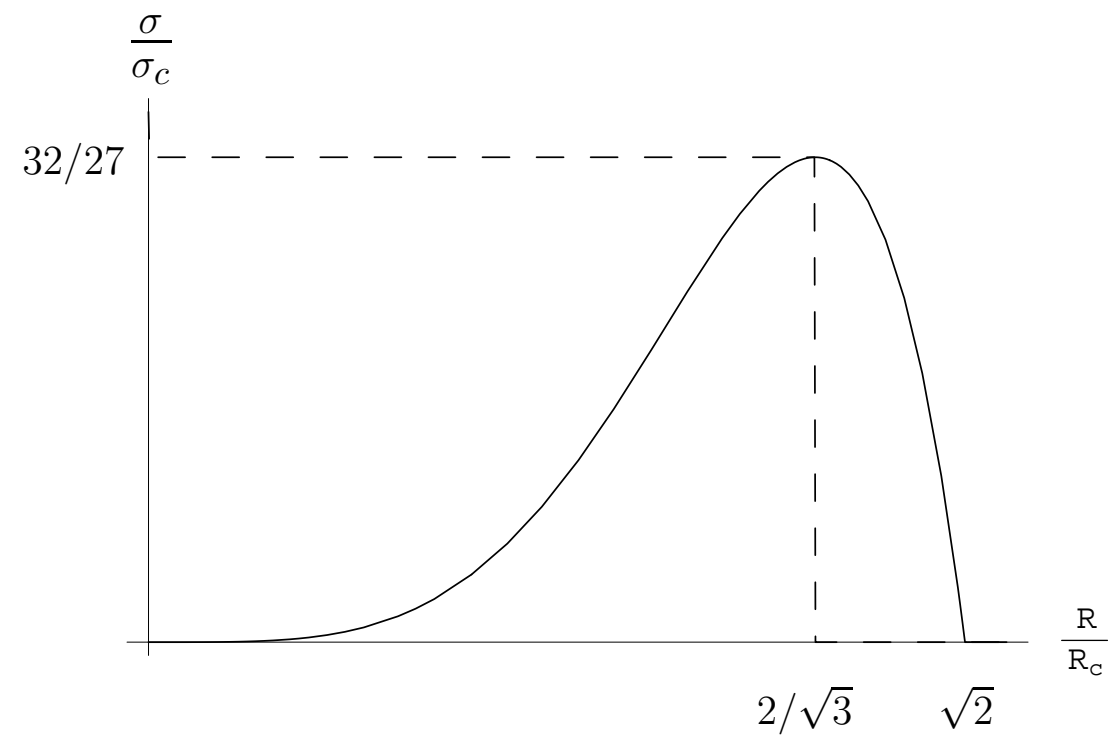

FIG. 1. The conductivity profile on the middle-plane $z=0$ described by Eq. (32) 


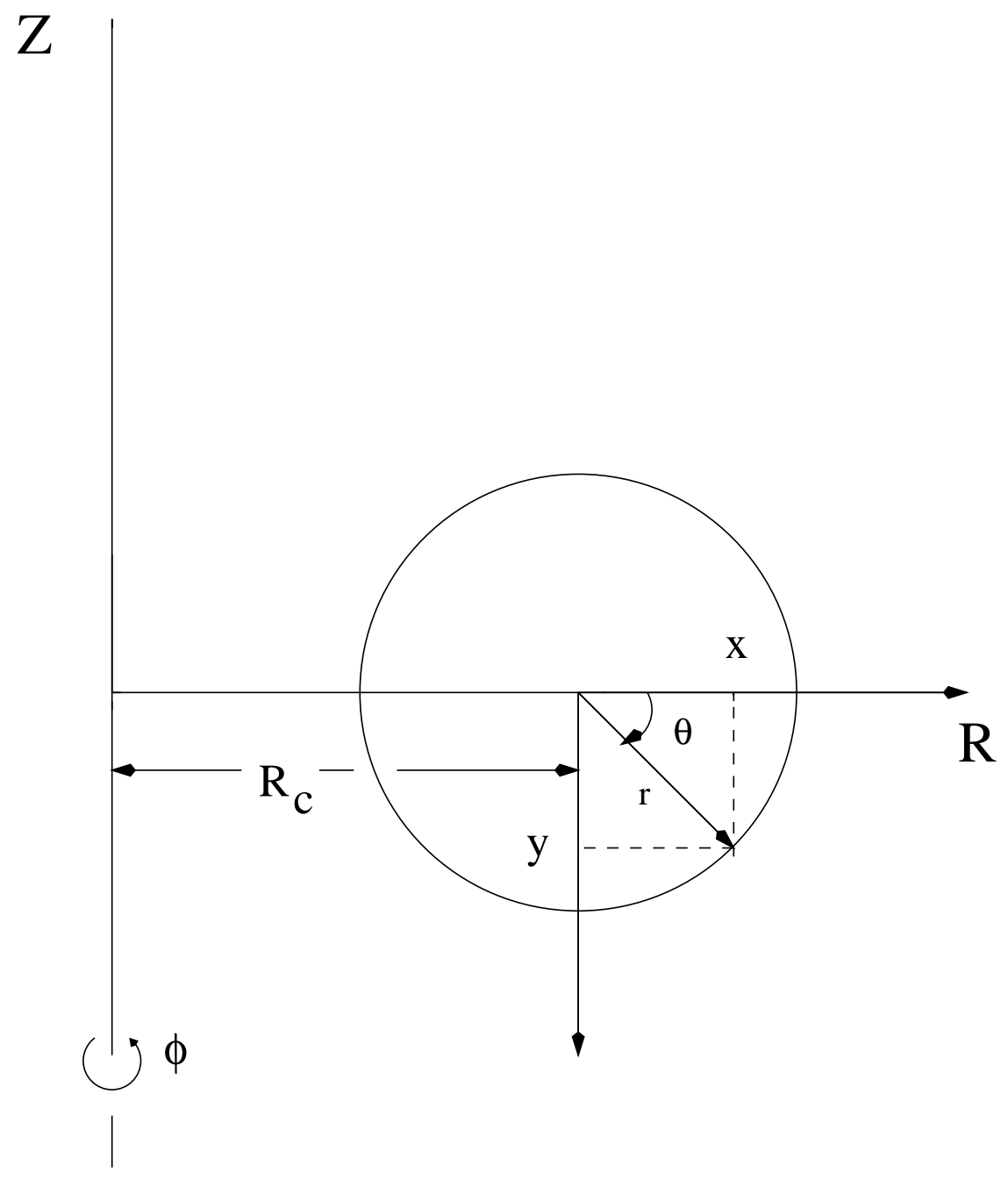

FIG. 2. The system of coordinates $(x, y, \phi)$. 